\title{
Overtone Mobility Spectrometry: Part 2. Theoretical Considerations of Resolving Power
}

\author{
Stephen J. Valentine, Sarah T. Stokes, Ruwan T. Kurulugama, \\ Fabiane M. Nachtigall, and David E. Clemmer \\ Department of Chemistry, Indiana University, Bloomington, Indiana, USA
}

\begin{abstract}
The transport of ions through multiple drift regions is modeled to develop an equation that is useful for an understanding of the resolving power of an overtone mobility spectrometry (OMS) technique. It is found that resolving power is influenced by a number of experimental variables, including those that define ion mobility spectrometry (IMS) resolving power: drift field $(E)$, drift region length $(L)$, and buffer gas temperature $(T)$. However, unlike IMS, the resolving power of OMS is also influenced by the number of drift regions $(n)$, harmonic frequency value $(m)$, and the phase number $(\Phi)$ of the applied drift field. The OMS resolving power dependence upon the new OMS variables $(n, m$, and $\Phi)$ scales differently than the square root dependence of the $E, L$, and $T$ variables in IMS. The results provide insight about optimal instrumental design and operation. (J Am Soc Mass Spectrom 2009, 20, 738-750) (C) 2009 American Society for Mass Spectrometry
\end{abstract}

W hen a pulse of ions is injected into a buffer gas, different species separate under the influence of an electric field because of differences in their mobilities through the buffer gas [1-4]. This phenomenon is the basis for a widely used analytical technique for resolving different components of mixtures called ion mobility spectrometry (IMS). The ability to isolate different species can be understood by considering the resolving power $\left(t_{d} / \Delta t\right)$, where $t_{d}$ is the time required for ions to drift through the buffer gas and $\Delta t$ corresponds to the full width at half maximum (FWHM) of the packet upon exiting the drift region. In the limit of an infinitely narrow injection pulse, the resolving power can be expressed as the ratio of the total drift length $\left(L\right.$, or $\left.t_{d}\right)$ and the spatial width $(\Delta L$, or $\Delta t)$ ) of the ion packet exiting the drift tube [5]. The Einstein relation between the mobility $(K)$ and the diffusion coefficient $(D)$ (i.e., $D=\sqrt{k_{b} T K l e}$, where, $e$ and $k_{b}$ correspond to the elementary charge and Boltzmann's constant and $T$ is the buffer gas temperature) can be used to estimate $\Delta L$ based upon ion displacement leading to an expression for the IMS resolving power $\left(R_{I M S}\right)$, eq $1,[1,5,6]$

$$
\frac{t}{\triangle t}=\left(\frac{E e L}{16 k_{b} T \ln 2}\right)^{1 / 2}
$$

Of note is the dependence of resolving power on the square root of the various parameters. This relationship imposes limits on the ultimate instrument performance. For example, doubling $L$ does not double the resolving

Address reprint requests to Dr. D. E. Clemmer, Department of Chemistry, Indiana University, 800 E. Kirkwood Ave., Bloomington, IN 47405, USA. E-mail: clemmer@indiana.edu power; rather, a 2-fold increase in $L$ (holding $T$ and $E$ constant) results in only $\sim 40 \%$ increase in resolving power.

In the present paper, we report modeling studies of ion transport through multiple drift regions to which the drift fields are applied at varying frequencies, the experimental setup used in overtone mobility spectrometry (OMS). The understanding that is gained from modeling allows us to develop a simple equation that can be used to estimate the OMS resolving power $\left(R_{O M S}\right)$. The equation describing the OMS resolving power accounts for a number of geometrical OMS device configurations as well as those parameters used to define $R_{I M S}$. However, these studies indicate that variation of the parameters which define $R_{I M S}(E, L$, and $T$ ) have only a limited impact on the $R_{O M S}$. Instead, the factors having the greatest influence on $R_{O M S}$ appear to be the number of phases for the system (i.e., the number of unique drift field application settings as well as the number of drift regions in a complete ion transmission/ elimination cycle, see below for complete description), the overall number of ion drift regions, and the drift field setting frequency (overtone number). A surprising result is the unit proportionality relationship between $R_{O M S}$ and the number of drift regions (in effect $L$ ) as well as the frequency suggesting the ability to garner much improved instrument performance (with respect to resolution) for proportionate changes when compared with IMS techniques.

It is important to note that the comparisons to $R_{I M S}$ (with respect to $L$ ) described here are based on the use of a constant drift field. Equation 1 can be rewritten such that the product of $L$ and $E$ is denoted as the drift voltage $(V)$. Extensive work has shown that increased resolving power can be obtained by optimizing $V$ and 
the dependence on drift tube length has been described in detail [7]. However, because of the interest in retaining structural information (which can assist ion identification efforts), the comparison has been carried out using low-field conditions which necessitate a square-root dependence on drift length as described herein.

There is currently considerable interest in developing and improving mobility based separation methods. In addition to efforts to improve IMS techniques, [8-13] a new method called field asymmetric (FA)IMS has been developed [14-22] Additionally, differential mobility analysis (DMA) [23, 24], which has traditionally been used for sizing of particles, [23, 25] has been extended for analysis of large molecular systems (such as polymers, proteins and protein complexes) [23, 24]. The present paper adds to theoretical work associated with understanding these mobility-based techniques.

\section{Methods}

\section{Experimental: General Description of OMS}

It is useful to begin by describing the OMS separation process. For convenience, Table 1 provides a summary of all variable definitions used in this paper. Since IMS techniques, including descriptions of a range of instruments [26-41] and theoretical treatments [1, 6, 42-46], are described elsewhere, further discussion of the various techniques is not provided here.

Each OMS instrument contains a specified number $(n)$ of sequential drift regions $(d)$ containing both ion transmission $\left(d_{t}\right)$ and ion elimination $\left(d_{e}\right)$ regions. In the experimental designs explored so far, the $d_{e}$ regions constitute a small portion of each $d$ region and are bounded by two electrostatic grids to help maintain drift field uniformity. A dc field is applied across the $d_{t}$ regions at a defined frequency. This application creates a frequency-dependent repulsive field in some of the $d_{e}$ regions, and this leads to elimination of portions of the continuous ion beam. It is the frequency of, and the order in which, this repulsive field is applied to the $d_{e}$ regions of the OMS device that serves to transmit ions of specific mobilities. To illustrate the overall process of OMS measurements, we present a description of several instrumental setups and provide examples of experimental data.

\section{Experimental: OMS Instrument Mode of Operation}

The mode of operation of an OMS instrument is determined by the number of phases or drift field application settings. This number is denoted by $\Phi$. Hereafter, $\Phi$ represents a positive integer indicating the method of applying drift field pulses to transmit ions of specific mobilities through the OMS device. Figure 1 illustrates
Table 1. Definitions for variables and descriptive terms

\begin{tabular}{|c|c|}
\hline Variable & Definition \\
\hline $\bar{D}$ & Diffusion coefficient \\
\hline$k_{b}$ & Boltzmann's constant \\
\hline$T$ & Temperature \\
\hline$K$ & Ion mobility \\
\hline$e$ & Elementary charge \\
\hline$t_{a}$ & Measured drift time \\
\hline$\Delta t$ & $\begin{array}{l}\text { Width at half maximum of intensity peak (in } \\
\text { time) }\end{array}$ \\
\hline$E$ & Drift field \\
\hline$L$ & Drift region total length \\
\hline$\Delta L$ & $\begin{array}{l}\text { Width at half maximum of intensity peak (in } \\
\text { length) }\end{array}$ \\
\hline$R_{I M S}$ & Resolving power for IMS \\
\hline$R_{O M S}$ & Resolving power for OMS \\
\hline$d$ & OMS drift region segment \\
\hline$d_{t}$ & Ion transmission region of $d$ \\
\hline$I_{t}$ & Length of the ion transmission region of $d$ \\
\hline$d_{e}$ & Ion elimination region of $d$ \\
\hline$l_{e}$ & Length of the ion elimination region of $d$ \\
\hline$n$ & $\begin{array}{l}\text { Number of } d \text { regions in OMS separation } \\
\text { device (positive integers) }\end{array}$ \\
\hline$\Phi$ & OMS system phase (positive integers) \\
\hline$m$ & $\begin{array}{l}\text { Harmonic frequency number (determined } \\
\text { by } \Phi \text { and } h \text { ) }\end{array}$ \\
\hline$j$ & $\begin{array}{l}\text { Index for field array regions in ion trajectory } \\
\text { simulations (zero and positive integers) }\end{array}$ \\
\hline$h$ & $\begin{array}{l}\text { Index to relate OMS phase and harmonic } \\
\text { frequency (positive integers) }\end{array}$ \\
\hline$\sqrt{\overline{r^{2}}}$ & $\begin{array}{l}\text { Root mean square ion displacement due to } \\
\text { mobility }\end{array}$ \\
\hline$\theta$ & $\begin{array}{l}\text { Randomization angle for ion trajectory } \\
\text { simulations }\end{array}$ \\
\hline$P_{w 1}$ & $\begin{array}{l}\text { Pulse width corresponding with the OMS } \\
\text { peak apex frequency setting }\end{array}$ \\
\hline$P_{w 2}$ & $\begin{array}{l}\text { Pulse width corresponding with the base of } \\
\text { the OMS peak frequency setting }\end{array}$ \\
\hline$f_{f}$ & $\begin{array}{l}\text { Fundamental frequency determined from } \\
\text { the time required for an ion to traverse a } \\
\text { single } d \text { region }\end{array}$ \\
\hline$f_{m}$ & Harmonic field application frequency setting \\
\hline$f_{1}$ & $\begin{array}{l}\text { Frequency setting corresponding with the } \\
\text { apex of the OMS peak }\end{array}$ \\
\hline$f_{2}$ & $\begin{array}{l}\text { Frequency setting corresponding with the } \\
\text { base of the OMS peak }\end{array}$ \\
\hline$\Delta f_{i-W H M}$ & $\begin{array}{l}\text { Full width at half maximum peak height of } \\
\text { the OMS peak }\end{array}$ \\
\hline$v_{\text {dif }}$ & $\begin{array}{l}\text { Velocity due to ion diffusion, in this case } \\
\text { directed against mobility (see } \\
\text { Supplementary Information) }\end{array}$ \\
\hline$C_{2}$ & $\begin{array}{l}\text { A constant associated with converting the } \\
\text { diffusion term in the } R_{\text {OMS }} \text { equation with } \\
R_{\text {IMS. }} C \sim 0.74 \text {. }\end{array}$ \\
\hline$\Omega$ & Ion collision cross section \\
\hline$P$ & Buffer gas pressure \\
\hline$m_{1}$ & Mass of the ion \\
\hline$m_{B}$ & Mass of the buffer gas \\
\hline$N$ & $\begin{array}{l}\text { Neutral number density under STP } \\
\text { conditions }\end{array}$ \\
\hline
\end{tabular}

a portion of an OMS drift region; eight consecutive $d_{t}$ regions with accompanying $d_{e}$ regions are shown. Also shown in Figure 1 are electric field conditions for a 

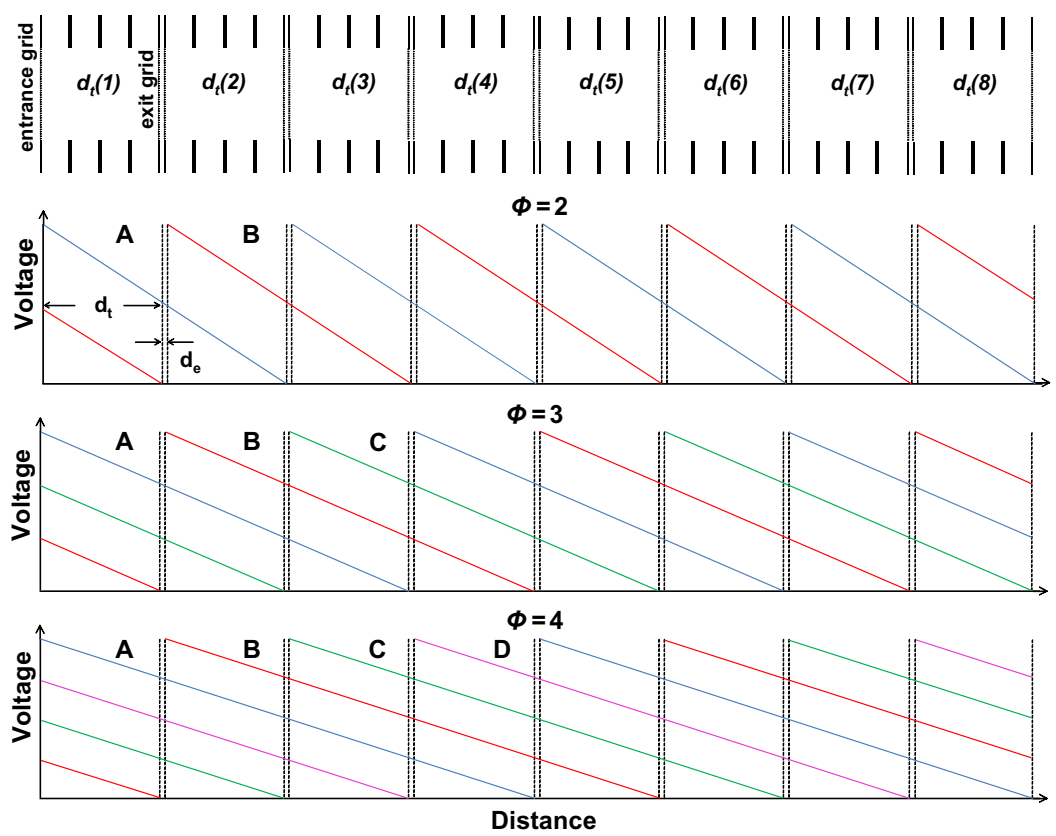

\begin{abstract}
Figure 1. Illustration of the drift regions of an OMS device. Shown are eight $d$ regions (containing both $d_{t}$ and $d_{e}$ sections). Also shown are the field modulation settings for OMS experiments utilizing phase conditions of $\Phi=2,3$, and 4. For the two-phase system, the blue and red traces correspond to voltage settings $A$ and $B$ respectively. For the three-phase system, the blue, red, and green traces correspond with the voltage settings $\mathrm{A}, \mathrm{B}$, and $\mathrm{C}$ respectively. Finally, for the four-phase system, the blue, red, green, and pink traces correspond with the voltage settings A, B, C, and D.
\end{abstract}

two-phase, three-phase, and four-phase system. First consider the operation of a two-phase OMS separation. Here, the drift field settings are modulated between two different settings. As shown in Figure 1, the first field setting (A) consists of multiple regions (each $2 d$ long) the first commencing with the gridded lens immediately preceding $d_{t}(1)$ and extending to the first gridded lens of the $d_{e}(2)$ region. At the same time a uniform field is applied to subsequent regions of equal length [e.g., fields across the $d_{t}(3)$ to $d_{e}(4)$ regions, the $d_{t}(5)$ to $d_{e}(6)$ regions, and the $d_{t}(7)$ to $d_{e}(8)$ regions]. This field setting creates repulsive fields in the ion elimination regions $d_{e}(2)$, $d_{e}(4), d_{e}(6)$, and $d_{e}(8)$. The second field setting (B, in Figure $1)$ shifts the multiple field regions by one $d$ region, and thus the ion elimination regions now become $d_{e}(1), d_{e}(3)$, $d_{e}(5)$, and $d_{e}(7)$. A complete ion transmission/elimination cycle shifts from one setting to the other and then back again (e.g., $\mathrm{A} \rightarrow \mathrm{B} \rightarrow \mathrm{A}$ for the two-phase system in Figure 1).

To understand the consequences of the field modulation consider the transmission of ions from a continuous ion beam using a two-phase OMS approach, a portion of the continuous ion beam fills the $d_{t}(1)$ region (Figure 1). Under the first field settings (A in Figure 1), ions are allowed to pass through the $d_{e}(1)$ region into the $d_{t}(2)$ region. Then the field is modulated to that of the second setting and the $d_{e}(2)$ region is set to transmit ions while the $d_{e}(1)$ region is set to eliminate ions. Thus, ions in the $d_{t}(2)$ region are transmitted to the $d_{t}(3)$ region, however, the ions that subsequently filled the $d_{t}(1)$ region are eliminated in the $d_{e}(1)$ region. This gives rise to an overall $50 \%$ duty cycle for the two-phase OMS system. That is, every other portion of the ion beam filling the $d_{t}(1)$ region is transmitted down the axis of the OMS device. Transmission of ions in this fashion is only possible if the mobilities of the ions are in resonance with the alternating field settings. That is, ions must have mobilities allowing them to traverse exactly one $d$ region in one field application. Ions with mobilities that are off resonance either traversing the $d$ region too quickly or too slowly are eventually eliminated in one of the $d_{e}$ regions. This applied frequency for ions having resonant mobilities is termed the fundamental frequency $\left(f_{f}\right)$.

Having described the field settings for a two-phase system, it is possible to depict the modulation process for a three-phase and a four-phase system. Again the number of distinct field settings is equal to $\Phi$. Additonally, the number of adjacent $d$ regions across which the field is applied is also equal to $\Phi$ and therefore a complete transmission/elimination cycle is equal to $\Phi$ field settings. To understand the impact on ion transmission with increasing $\Phi$ it is instructive to consider operation with a greater number of phases as shown in Figure 1. Here, we consider the four-phase system. As the elimination pulse moves from $d_{e}(1)$ to $d_{e}(2)$, ions from the $d_{t}(1)$ region fill the $d_{t}(2)$ region. The subsequent field application results in a difference compared with the two-phase operation. Rather than shift back to $d_{e}(1)$, the elimination pulse moves to $d_{e}(3)$ and ions from the $d_{t}(2)$ region move into the $d_{t}(3)$ region. Additionally, ions from the $d_{t}(1)$ region move into the $d_{t}(2)$ region. This proceeds until ions occupy the $d_{t}(1), d_{t}(2), d_{t}(3)$, and 
$d_{t}(4)$ regions. At that time, the elimination pulse shifts back to the $d_{e}(1)$ and ions in the $d_{t}(1)$ region are eliminated while ions in the other three regions are transmitted throughout the OMS device. Thus a fourphase system has a duty cycle of $75 \%$. Overall, this discussion leads to a general expression for the duty cycle (neglecting ion diffusion) of an OMS device as a function of phase number (i.e., $1-1 / \phi$ ).

\section{Experimentally Recorded OMS Distributions for Different Values of $\Phi$}

OMS distributions can be obtained by recording the ion signal for a specific ion at given field application frequencies. Figure 2 shows the OMS distributions obtained for the $[\mathrm{M}+\mathrm{Na}]^{+}$ion for the oligosaccharide raffinose. The data have been recorded over a range of OMS system phase settings $(\Phi=2$ to 6$)$. For each setting for $\Phi$ a dominant peak corresponding to the fundamental frequency is observed. This peak is observed to broaden with increasing $\Phi$. In addition to the fundamental frequency peak, a number of other peaks are observed for each OMS separation. For example, for the analysis that utilized the two-phase system, peaks

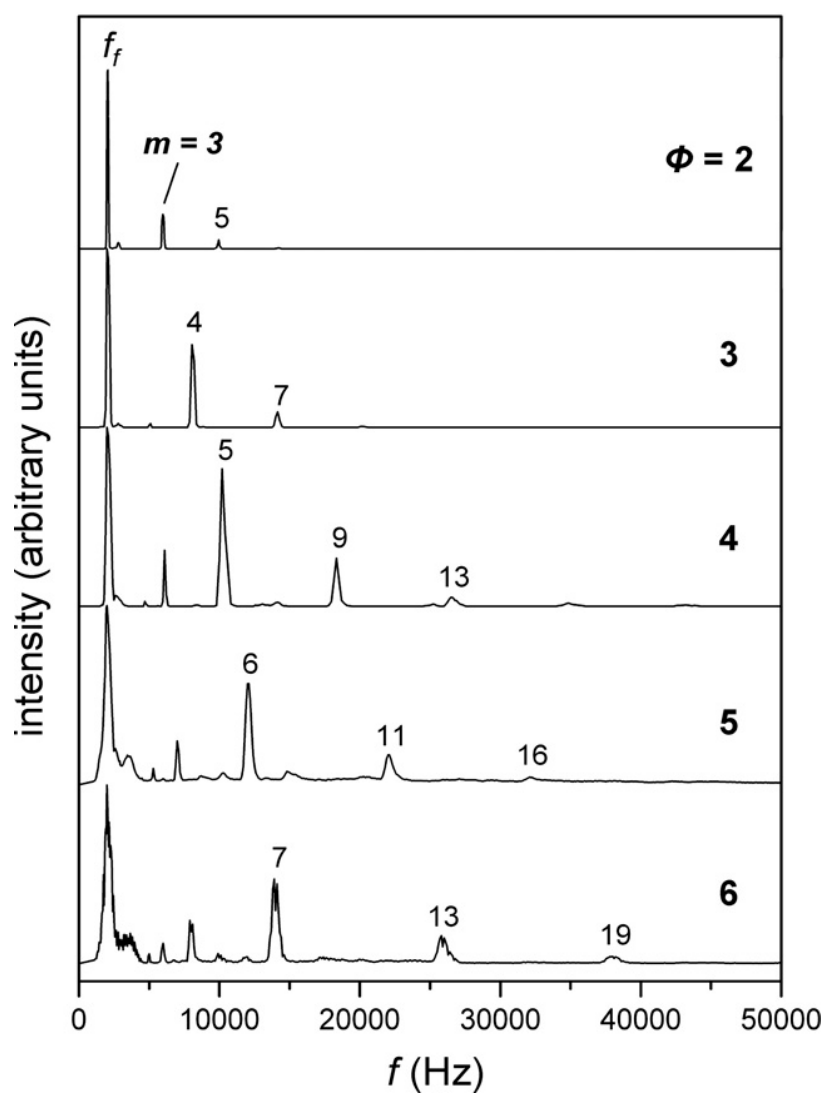

Figure 2. Experimental OMS distributions for the $[\mathrm{M}+\mathrm{Na}]^{+}$ion of raffinose (a three residue oligosaccharide) obtained upon electrospray ionization. The data are plotted as a function of $\Phi$ with the plots (top to bottom) representing a range of $\Phi=2$ to 6 . Also depicted are the $m$ values for specific overtone frequencies that permit ion transmission. are observed at frequencies that are exact multiples of $f_{f}$ (including $3 \cdot f_{f}$ and $5 \cdot f_{f}$ ). The transmission of ions at "overtone" frequencies is also observed for other values of $\Phi$. From the experimental data in Figure 2 for raffinose ions (and other ions that show the same behavior), clearly the pattern of overtone peaks that are transmitted depends on the magnitude of $\Phi$. Hereafter, the number of the harmonic frequency associated with OMS distribution peaks is denoted by $m$ and is defined as $\Phi(h-1)+1$, where $h$ is a positive integer representing the OMS harmonic index. The harmonic frequency representing the field application frequency setting for ion transmission can thus be expressed as $f_{m}=m \cdot f_{f}$. To better understand the process by which ions are transmitted at overtone frequencies, two examples are presented below.

\section{Origin of Peaks at Overtone Frequencies}

The origin of overtone peaks can be understood by considering the hypothetical ion beam and transmitted ion distributions that are shown in Figure 3. Here we demonstrate the transmission of ions for the second overtone frequency ( $m=3$ harmonic) for a two-phase OMS separation. During the first field application, the forward most third of the ions in the $d_{t}(1)$ region are transmitted into the $d_{t}(2)$ region. The elimination field is then shifted to $d_{e}(1)$ and during this time the middle third of the ions in the $d_{t}(1)$ region is eliminated. Upon the next application, the final third of the ions in the $d_{t}(1)$ region is transmitted into the $d_{t}(2)$ region. In this manner, alternating batches of ions are transmitted through the OMS device still yielding a 50\% duty cycle (neglecting diffusion). As described in the manuscript published jointly with this work (experimental portion), resolving power increases with increasing $m$.

From Figure 3 and the discussion given above, we see that transmission of ions at overtone frequencies will also depend on $\Phi$. For example, for conditions in which the first overtone frequency is applied to a two-phase system, half of the ions in the $d_{t}(1)$ region [those nearest the $d_{e}(1)$ ] are transmitted into the $d_{t}(2)$ region. However, because $m$ is equal to 2 , the second half of the ions in the $d_{t}(1)$ region are eliminated in the $d_{e}(1)$ region. During this time, ions in the first half of the $d_{t}$ (2) region move to the second half of this region. However, elimination subsequently shifts to the $d_{e}(2)$ region and these ions are eliminated as well. Thus, for the harmonic frequency $m=2$ and OMS system phase setting $\Phi=2$, ion transmission is not allowed. For the two-phase system, observed harmonic frequencies include $m=1$ (fundamental), 3, and 5 (Figure 2). For a four-phase system observed harmonic frequencies include $m=1,5,9$, and 13 (Figure 2). From these observations and the above rationale, we determine the empirical relation that ions will be transmitted at multiples of the fundamental frequency (i.e., overtones), as mentioned above. Overall, these considerations explain 


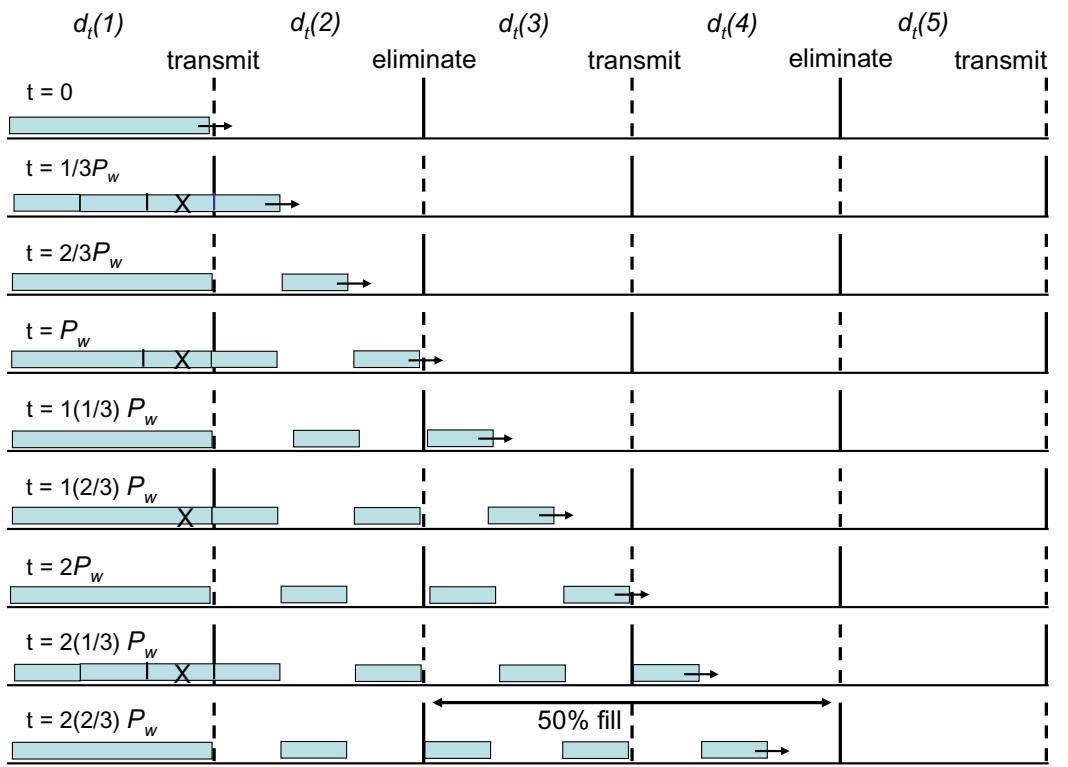

Figure 3. Illustration of the effect of overtone frequency on ion transmission. A continuous ion beam filling the $d_{t}(1)$ region followed by transmission of portions of the ion beam is shown. $d_{t}$ regions are indicated above each successive region and $d_{e}$ regions are denoted as either elimination or transmission. Also shown is the field application time $(t)$ as a function of the $f_{f}$ time $(T)$. $d_{e}$ regions alternate between elimination (gates) and transmission (grids) conditions. This illustration shows a two-phase system where two field settings are required to complete a transmission/elimination cycle. The $m=3$ harmonic frequency (second overtone) is represented here.

the pattern of peaks that is observed experimentally for different phase values for raffinose (Figure 2).

Because of the relationship between $\Phi$ and $m$ (via $h$ ), the focus of the current work is the derivation of an expression for resolving power for those features representing the fundamental and overtone peaks for the different phase systems. These are the major peaks labeled in Figure 2. Here we note that a number of other peaks are observed, most noticeably between the fundamental frequency peak and the $h=1$ overtone peak for different values of $\Phi$ (particularly for greater values). The origin of these peaks is still under investigation and as such they are not treated here in terms of estimation of resolving power. Rather, investigations focus on the dominant peaks (Figure 2) and their relationship to resolving power.

\section{Ion Trajectory Simulations}

To better understand the separation process of OMS, ion trajectory simulations have been performed. We have used a field array containing $11 d$ regions. The algorithm used to perform the simulations has been written in house and is conceptually similar to those described previously [47, 48]. Briefly, the calculations utilize two-dimensional field arrays similar to those generated in SIMION [49] to determine time-dependent ion displacements. The displacement calculation of an ion is the sum of the ion motion contributions from the mobility of the ion $(K)$ as well as its diffusion. The former contribution is field-dependent [i.e., the drift velocity is proportional to the product of the mobility and the electric field $(K E)]$ [1], whereas the latter is a randomized contribution in the algorithm (discussed below). Although the algorithm is similar to that described previously [48], the field arrays of two dimensions are utilized as opposed to three. This change is made because it reduces the time required to model trajectories of thousands of ions. The two dimensions (instead of three) utilized include the ion axis (z-axis) as well as the height (y-axis). Two-dimensional ion trajectory simulations performed in SIMION [49] utilize the same field array dimensions.

The modeling algorithm also allows different OMS phase systems to be examined. For simplicity, trajectory simulations for a two-phase system are described below. The modeling requires two field arrays including one in which the $1+2 j(j=0,1,2,3,4,5$, and 6$) d_{e}$ regions (see above) contain fields that will transmit ions; the remaining $d_{e}$ regions contain fields that will cause the elimination of ions. For the second field array, the transmit and eliminate $d_{e}$ regions are reversed. Initially the algorithm selects one field array and reads in a region (four field array points) that flanks the two-dimensional position of the ions. A weightedaverage field is then calculated for both dimensions based on the distance of the ion to the field values provided at each array point. The displacement due to the ion mobility is then calculated for a user-defined time step (typically $\leq 2 \mu \mathrm{s}$ ) using the drift velocity $(K E)$. 
Displacement of ions due to diffusion is simulated by

$$
\sqrt{\overline{r^{2}}}=(4 D \Delta t)^{1 / 2}
$$

where, $\sqrt{\overline{\overline{r^{2}}}}$ is the root mean-square displacement of the ion for time increment $\Delta t$ and ion diffusion coefficients can be obtained as mentioned above. For ions used in the simulations, $K$ is taken from an experimentally available value (e.g., the mobility of singly-charged bradykinin). To simulate random diffusion in two dimensions, $\sqrt{\overline{r^{2}}}$ is converted into a polar coordinate vector by randomizing a single angle $(\theta)$. The $y$ - and $z$-axis vector components are determined from $\sqrt{\bar{r}^{2}} \cdot \sin \theta$ and $\sqrt{\overline{r^{2}}} \cdot \cos \theta$, respectively. The diffusion value is then added to the two-dimensional mobility calculation to provide a net ion displacement for each time increment.

To perform the trajectory simulation of ions in the two-phase system, a model has been devised where ion origin is referenced with respect to the $d_{t}(1)$ region immediately preceding the first ion gate $\left[d_{e}(1)\right]$. It is important to note that $d_{t}(1)$ is equal in size to the other $d_{t}(n)$ regions $(n=2$ to 11$)$. Because the duty cycle of a two-phase system is $50 \%$ (neglecting diffusion, see above), it is only necessary to determine the trajectories of ions as a function of position within this initial $d_{t}(1)$ region. For the ion trajectory simulations reported here, the $d_{t}(1)$ region constitutes the first 80 grid units $(\sim 5.08$ $\mathrm{cm}$ ) of each field array file. Subsequent $d_{t}$ regions are of the same length and the conjoining $d_{e}$ regions are each $\sim 0.32 \mathrm{~cm}$. For the simulations described here, the trajectories for 20 theoretical ions at each location of origin (grid unit) have been simulated. Thus a complete analysis for a specific frequency requires 1600 ion trajectory simulations.

\section{Results and Discussion}

\section{Ion Trajectory Simulations}

The trajectory simulations described above have been performed as a function of frequency to provide the peak profiles shown in Figure 4. Each of 10 to 20 incremental frequencies spanning the fundamental frequency as well as the overtones with harmonic frequencies of $m=3$ and 5 is used. The modeling peak profiles resemble the experimental results on several fronts. First the relative peak heights, which are approximately inversely correlated with $m$, are similar to experimentally observed peak heights. Second, the relative resolving powers for the modeled two-phase system [consisting of $11 d$ regions] are similar to values obtained for peptide ions. That is, resolving powers of $\sim 12, \sim 28$, and $\sim 46$ are calculated for the $m=1,3$, and 5 frequencies, respectively. This increase in resolving power with increasing overtones is similar to that observed experimentally.

It is instructive to consider which ions in the $d_{t}(1)$ region are transmitted through the entire mobility de-

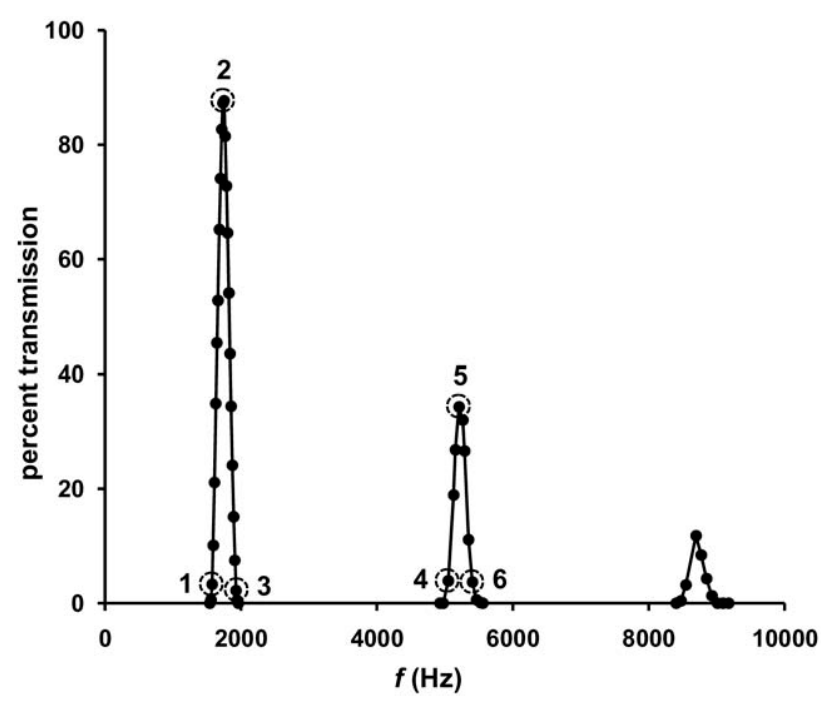

Figure 4. OMS peak profiles obtained from ion trajectory simulations. Simulations have been performed for an OMS approach utilizing $\Phi=2$ and $n=11$. Peaks have been modeled for the fundamental frequency as well as the $m=3$ and $m=5$ harmonic frequencies (second and fourth overtones). Dashed-line circles are used to indicate the ion transmission profiles (Supplementary Figures 1 and 2 in the Supplementary Information section) of ions in the $d_{t}(1)$ region (Figures 1 and 3 ) for the given frequency (see text for details).

vice as determined by the ion trajectory simulations. For discussion purposes, the $d_{t}(1)$ region has been divided into 80 initial ion positions and three field application frequencies are considered. Frequencies 1 and 3 (Figure 4) correspond to the left and right, respectively, base of the peak while frequency 2 corresponds to the peak apex (fundamental frequency). For conditions using frequency 1 , only ions with initial positions furthest from the $d_{e}(1)$ region [positions of $\sim 1$ to 10 in the $d_{t}(1)$ region] are effectively transmitted through the OMS device. All other ions are effectively eliminated. For frequency 2, transmission is largely ensured for ions with starting $d_{t}(1)$ region positions of $\sim 20$ to 60 although ion transmission drops off significantly for the far left and right initial ion positions of the $d_{t}(1)$ region. This is due to the ion diffusion component of the ion trajectory modeling. That is, ions originating in these areas can access preceding or trailing $d_{e}$ regions for which ion elimination settings are employed. Overall, for frequency 2 , the vast majority of the ions in $d_{t}(1)$ is transmitted through all ion gates and reaches the end of the two-phase OMS device. Frequency 3 produces a transmission profile very similar to frequency 1 with the exception that now the only ions that are transmitted through the instrument are those closest to the $d_{e}(1)$ region [i.e., those with high initial $d_{t}(1)$ positions, $\sim 70$ to 80]. Examination of such transmission profiles is important because it provides insight necessary for the development of an analytical expression for resolving power (see below). The transmission profile described above is graphically represented as Supplementary Figure 1 in the Supplementary Discussion section, which can be found in the electronic version of this article. 
It is also useful to consider the transmission of ions for a single overtone frequency setting. At the second overtone frequency $(m=3)$, ions are transmitted in the left and right third of the $d_{t}(1)$ region as shown in Figure 3 . Ions in the middle third of the region are eliminated as described above. It is important to note that the smaller transmitted beam portions behave similarly to the larger transmitted portions for the fundamental frequency. That is, it is useful to consider the initial positions of the transmitted ions at the peak base frequencies (frequencies 4 and 6 in Figure 4) as well as the $m=3$ harmonic (frequency 5 in Figure 4). Using the 80 divisions again for initial starting positions in the $d_{t}(1)$ region, ions furthest from and closest to the $d_{e}(1)$ region are transmitted using frequencies 4 and 6 , respectively. This occurs for both the front third and the last third of the transmitted $d_{t}(1)$ region ions. Because of this behavior, it is possible to elucidate a single expression for resolving power that also takes into account overtone frequency transmission. As before, the transmission profile for the $m=3$ harmonic frequency is shown as a graph (Supplementary Figure 2) in the Supplementary Discussion section.

\section{Developing an Analytical Expression for Estimating Resolving Power}

To develop an expression for $R_{O M S}$, consider results from ion trajectory simulations. Of interest is the peak shape produced by the OMS method. By removing the random diffusion component from the ion displacement calculation in ion trajectory simulations (see above), it is possible to construct a peak shape that depends only on mobility filtering. Peaks produced by measuring ion transmission as a function of frequency (in the $f_{f}$ region) are triangular. As depicted in Supplementary Figure 1, when the frequency increases in the $f_{f}$ region, the total number of transmitted ions increases in a linear fashion until it reaches a maximum at $f_{f}$. From that point, the total number of transmitted ions decreases linearly with increasing frequency until reaching the base of the peak ultimately generating the equilateral triangle peak shape. When the triangular shaped peak is compared with the peak obtained from ion trajectory simulations (i.e., that accounting for diffusion as observed in Figure 4), a slight degree of broadening and apex curvature is observed; the $f_{f}$ peak shown in Figure 4 is $<2 \%$ broader than the triangular shape generated in the absence of diffusion. Of interest is the fact that diffusion plays little role in the overall peak width; this is captured in the $R_{O M S}$ expression as described below.

An expression for $R_{O M S}$ can be obtained by considering the particular ions that limit the overall resolving power. For example, at the base of the $f_{f}$ peak (frequency 1 in Figure 4), only a narrow range of ions [i.e., those furthest from the $d_{e}(1)$ region] is transmitted through the OMS device. Using this observation it is possible to construct a model from which an equation for resolving power can be derived. The model calls for the determination of the difference from the center of the OMS peak (frequency 2 in Figure 4) to the base of the peak (frequency 1). This difference can then be used to estimate the peak width at half height $\left(\Delta f_{F W H M}\right)$. Because the true peak shape should be very similar to that of an equilateral triangle, this difference would represent [1/2] of the peak base, or the actual $\Delta f_{F W H M}$ by definition. From here, it is straightforward to determine the resolving power $\left(R=f_{f} / \Delta f_{F W H M}\right)$.

We begin by considering a peak that is associated with ions that are transmitted when the drift field frequency is set to $\sim 1 / t_{d}$ (i.e., the resonant frequency, $f_{f}$, for the ions). It is important to note that while ions are optimally transmitted at the resonance frequency, one sees that some ions are also transmitted at slightly lower and higher frequencies, giving rise to the rising and falling edges, respectively, of the peak (shown in Figure 4). The origin of the off-resonance portions of the peak can be understood by considering the initial ion position within the $d_{t}(1)$ region. Neglecting diffusion, when the ion's mobility matches to the applied frequency, ions at any position in the $d_{t}(1)$ region will be transmitted to the $d_{t}(2)$ region. Moreover, at this resonant frequency condition, the ions will occupy the same position in the $d_{t}(2)$ region after one drift field application setting. An ion starting at position 1 (see Supplementary Figure 1) in the $d_{t}(1)$ region will arrive at position 1 of $d_{t}(2)$. To reach the last elimination region, $d_{e}(n)$, will require $n$ field application settings $(n / 2$ transmission and elimination cycles for a two-phase system).

Now consider conditions where the applied frequency is lower than the ion's resonant fundamental frequency (frequency 1 in Figure 4). With each pulse all ions arrive at a position that is slightly more displaced within the next $d$ region (e.g., for the ion described above, position numbers of 10 and 20 in the $d_{t}(2)$ and $d_{t}(3)$ regions, respectively). An extreme case would be those ions having the greatest mismatch in mobility frequency and gating frequency. For the ion described above (i.e., starting at position 1) to be transmitted, it would need to reach the last $d_{e}$ region just before the drift field was switched from elimination to transmission (i.e., requiring one fewer or $n-1$ field application settings). This represents the worst case scenario; if the frequency/mobility mismatch were any greater (lower frequency field application), then the switch from elimination to transmission would occur at a longer time and the ion would have been eliminated at $d_{e}(n)$. In the limit of an infinitely small $d_{e}$ region, it can be argued that for the fundamental frequency setting, the ion would have experienced $n$ field application settings while for the frequency 1 setting (Figure 4) the ion would have experienced $n-1$ field application settings in the same time $\left(t_{\text {total }}=\right.$ time required to reach the $n^{\text {th }}$ gate). 
With an understanding that the time duration of a single field application setting $\left(P_{w}\right)$ is equal to $t_{\text {total }}$ divided by the total number of settings, we can obtain expressions for the field application time associated with the fundamental frequency $\left(f_{2}\right.$ in Figure 4$)$, as well as that associated with the base of the peak frequency $\left(f_{1}\right.$ in Figure 4). Equations for the former and latter are shown in eqs 3 and 4, respectively,

$$
\begin{aligned}
& P_{W 2}=\frac{t_{\text {total }}}{n}, \\
& P_{W 1}=\frac{t_{\text {total }}}{n-1} .
\end{aligned}
$$

These relationships can be used to relate the frequencies of $f_{1}$ and $f_{2}$ (see Supplementary Information) leading to eq 5 ,

$$
f_{1}=\frac{(n-1) f_{2}}{n} .
$$

Understanding that OMS peaks are triangular in nature and using eq 5, it is possible to obtain the simplified expression for ROMS shown in eq 6 (see Supplementary Information for equation derivation information details).

$$
R_{O M S}=n .
$$

Remarkably, the resolving power of the OMS technique for the fundamental peak depends only on the number of drift region segments. Although this is somewhat intuitive, it stands as an important result because it defines one important difference between OMS, where $R_{\text {OMS }}$ scales directly with $L$ (here $L$ is equal to $n$ multiplied by the length of the $d$ region), and IMS, where $R_{I M S}$ scales with the square root of $L$.

\section{Inclusion of Overtone Frequency Terms}

With an expression for the resolving power of the fundamental frequency, it is useful to include the effects of OMS system phase and harmonic frequency. Consider the case of the four-phase OMS device. Here, four ion gating regions are triggered sequentially to complete one transmission/elimination cycle. As described above, ions occupying the space of three $d$ regions are transmitted through the OMS device for every space equivalent to one $d$ region that leads to ion elimination (i.e., a duty cycle of $75 \%$ ). Because the total ion transmission range is three times larger than the comparable two-phase system (i.e., one with $d$ regions of identical length), the difference in number of pulses experienced by ions using field application frequencies $f_{1}$ and $f_{2}$ (Figure 4 ) is three. And thus the $R_{O M S}$ scales as $n / 3$ (see Supplementary Information for more details on this dependency). Although the signal level increases with increasing phase, the resolving power is lower by a factor of $(\Phi-1)^{-1}$ for a given value of $n$.
The opposite effect is observed with an increase in overtone. Consider the two-phase system operating at the $m=3$ harmonic frequency (second overtone). Because the overall frequency is three times greater than $f_{1}$, the total number of field applications experienced by an ion at the $m=3$ harmonic frequency transmission region is three times the number of $d$ regions or $3 n$. Ions at the base of the OMS peak profile (e.g., frequency 4 in Figure 4) will experience one fewer field application setting $(3 n-1)$ over the same time period. Thus, using the same derivation as that for eq 6 (see Supplementary Information), $R_{O M S}$ is observed to scale directly with the product of $m$ and $n$.

\section{Consideration of the Sizes of Transmission and Elimination Regions}

Until this point, the $d_{e}$ regions were treated as infinitely small. It is interesting to consider the consequences of different lengths of $d_{t}$ and $d_{e}$ regions (denoted $l_{t}$ and $l_{e}$ respectively). Consider the two-phase system. As mentioned above the difference in number of pulses experienced for ions at a field application frequency of $f_{1}$ versus a field application frequency of $f_{2}$ is $n-1$ and $n$, respectively. However, to be transmitted, over the same time period these ions are required to arrive just before (for the $f_{1}$ field application settings in Figure 4) and just after (for the $f_{2}$ field application settings in Figure 4) the last $d_{e}$ region. In these scenarios, the last $d_{e}$ region would be switching from eliminate to transmit and transmit to eliminate, respectively. These situations are only possible in the limit of an infinitely small $l_{e}$. Because $l_{e}$ is greater than zero, the $f_{1}: f_{2}$ ratio of $n-1: n$ (eq 5) should be revised. Because ions do not traverse the entire length of the last $d$ region for the $f_{1}$ field settings (compared with the $f_{2}$ settings), the difference in the number of field application settings is less than one. That is, the $n-1$ term can be corrected by a factor related to the change in distance traversed by the ions. The new term becomes $n-1\left[l_{t} / l_{t}+l_{e}\right]$. As noted above, this term is used to relate $f_{1}$ and $f_{2}$.

With an understanding of the influence of $n, \Phi, m$, as well as $l_{t}$ and $l_{e}$ on the relationship between $f_{1}$ and $f_{2}$, it is possible to obtain the following expression for $R_{O M S}$ :

$$
R_{\text {OMS }}=\frac{m n}{\phi-1-\frac{l_{e}}{l_{t}+l_{e}}} .
$$

This expression reveals several important factors affecting the overall resolving power. First, as observed above, $R_{\text {OMS }}$ increases linearly with increasing $n$. Similarly, $R_{O M S}$ increases linearly with $m$; that is, higher harmonic frequencies result in increased resolving power. However, the equation demonstrates that an increase in the number of phases of the system actually decreases the $R_{O M S}$. The final factor affecting $R_{O M S}$ is the ratio of $l_{e}$ to $l_{t}$. As this ratio increases, $R_{O M S}$ increases. 


\section{Inclusion of Diffusion}

It is important to note that eq 7 does not truly represent a worst-case scenario for the greatest mismatch in $f_{1}$ and $f_{2}$ since this expression does not include ion diffusion. The situation that would lead to the greatest influence on resolving power is if the ion diffusion is unidirectional. That is, net diffusion in the direction opposite the direction of mobility motion results in the greatest mismatch between $f_{1}$ and $f_{2}$. If an average velocity of diffusion $\left(v_{\text {dif }}\right)$ is subtracted from the drift velocity $(K E)$ used to calculate $R_{\text {OMS }}$ (see the Supplementary Information), $f_{1}$ is decreased, resulting in a wider base of the OMS peak. By introducing a diffusion component into the calculation it is possible to express $R_{O M S}$ in terms of $R_{I M S}$. The final $R_{O M S}$ equation accounting for all experimental parameters becomes,

$$
R_{\text {OMS }}=\frac{1}{1-\left[1-\frac{C_{2}}{R_{I M S}}\right]\left[\frac{m n-\left[\phi-1-\frac{l_{e}}{l_{t}+l_{e}}\right]}{m n}\right]},
$$

Here $C_{2}$ corresponds to a constant allowing the conversion to $R_{I M S}\left(C_{2} \approx 0.74\right)$.

Equation 8 provides insight about limits associated with diffusion. That is, in the limit of high $R_{I M S}$ (less diffusion), the denominator approaches a minimum value leading to a maximum $R_{O M S}$.

\section{Evaluation of the OMS Resolving Power Equation: Dependence on Number of Gating Regions and Applied Frequency}

We begin the evaluation of the expression for OMS resolving power by considering several experimental results. Figure 5 shows the experimental peak widths for the $[\mathrm{M}+\mathrm{Na}]^{+}$ion of the trisaccharide melezitose for varying numbers $(n)$ of $d$ regions. The data have been acquired by applying the fundamental frequency for a four-phase OMS device. The results show a substantial decrease in peak width with increasing $n$. At $n=11$, the measured FWHM is $\sim 1600 \mathrm{~Hz}$, whereas a value of $\sim 345 \mathrm{~Hz}$ is obtained at $n=43$. This large dependence on $n$ is intriguing because it suggests that OMS resolving power is not defined by a square root dependence on $L$ as is the case for IMS experiments. To evaluate the efficacy of the equation for predicting OMS resolving power as a function of $n$, calculated values have been compared with the experimental results obtained for the four-phase OMS system (Figure 5). The equation predicts a linear increase in $R_{O M S}$ from 3.6 to 13.0 at $n$-values of 11 and 43, respectively. A linear fit to the calculated values provides a line with a slope of 0.293 and an intercept of $0.471\left(R^{2}=0.999\right)$. The comparison shows that the trend of increasing resolving power with an increase in $n$ is observed for both the experimental

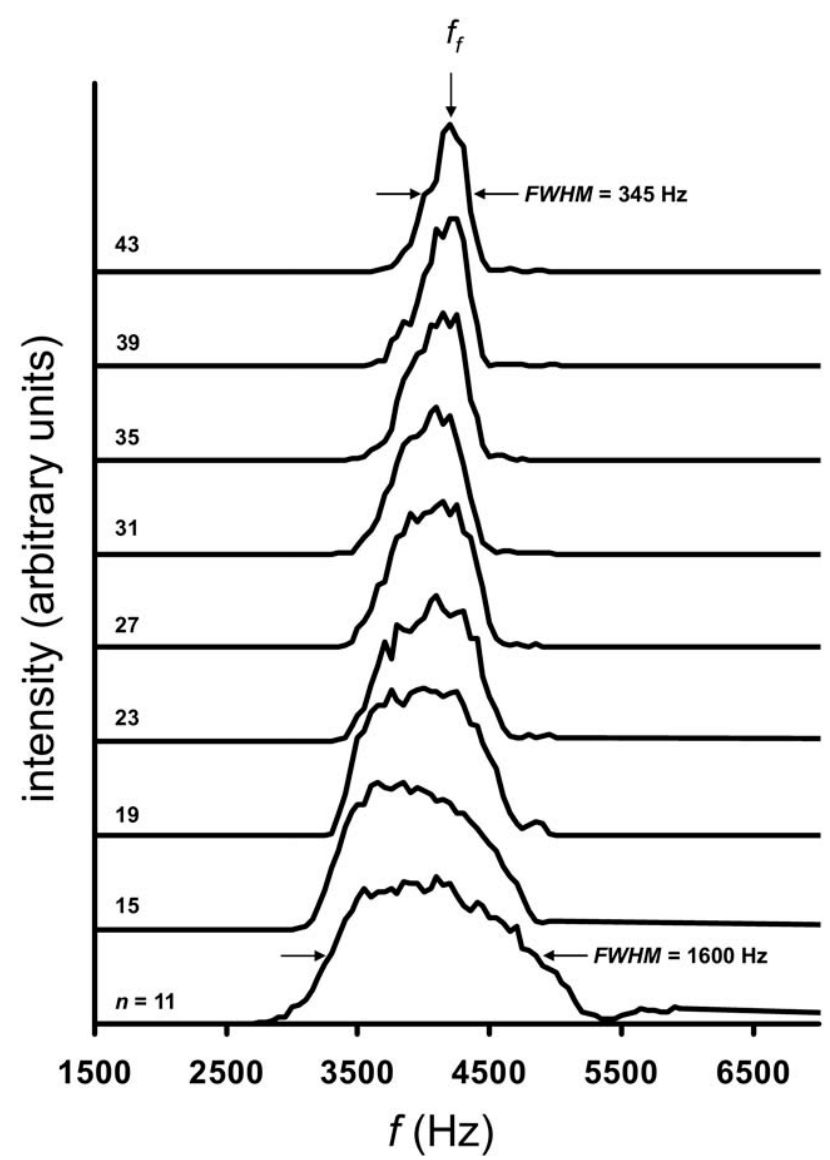

Figure 5. Experimental OMS distributions for the $[\mathrm{M}+\mathrm{Na}]^{+}$ion for the trisaccharide melezitose. The plots show a progression of OMS distributions as a function of $n$. The data span the range of that collected using $n=11 d$ regions (bottom trace) to $n=43 d$ regions (top trace). The position of $f_{f}$ is shown with an arrow. Additionally, the peak widths (FWHM) for the OMS distributions for $n=11$ and $n=43$ are noted. Here OMS conditions of $\Phi=4$ and $m=1$ are utilized. Other experimental conditions include $E=$ $10 \mathrm{~V} \cdot \mathrm{cm}^{-1}$ and $T=300 \mathrm{~K} \cdot l_{t}$ and $l_{e}$ region lengths of $2.83 \mathrm{~cm}$ and $0.24 \mathrm{~cm}$, respectively have been used in the experiment.

data and the calculated values. On average, the equation predicts a resolving power that is $\sim 37 \%$ higher than what is obtained experimentally. Calculated resolving power and experimentally determined values are shown as Supplementary Figure 3 in the Supplementary Information section.

The ability of the equation to predict resolving power as a function of the applied overtone frequency has also been investigated. The comparison has been evaluated using experimental data for three different systems ( $\Phi=2,3$, and 4). Additionally, the transmitted overtone peaks (for which $h=2$ to 3 ) of each system have been compared. The results of the comparison are shown in Figure 6. In general, the agreement is as observed before with the equation providing a resolving power that is $\sim 21 \%$ higher on average than experimental results for the fundamental frequency and the overtone frequencies (corresponding to $h=2$ to 3 ). For all systems, the trend of increasing resolving power 


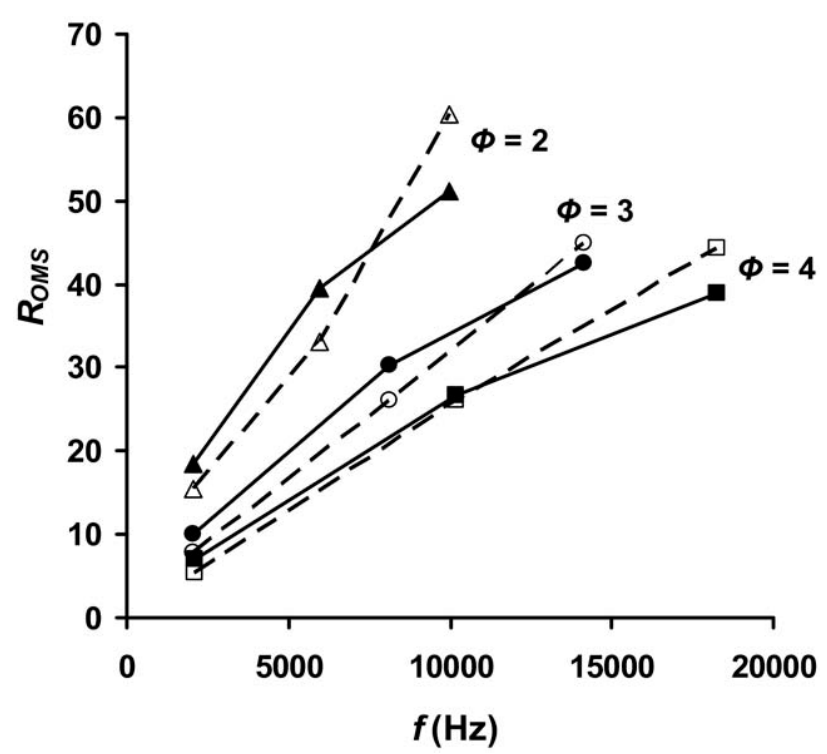

Figure 6. Plot of calculated and experimental $R_{O M S}$ dependence on the applied frequency for OMS approaches using $\Phi=2$ (triangles), $\Phi=3$ (circles), and $\Phi=4$ (squares). Solid symbols represent $R_{O M S}$ obtained from eq 20; open symbols represent experimental results for the respective values of $\Phi$. Only values for $h=1$ to 3 are shown for each value of $\Phi$. Conditions for the experiment and the calculation include $E=10 \mathrm{~V} \cdot \mathrm{cm}^{-1}$ and $T=$ $300 \mathrm{~K}$. Additionally, the values for $l_{t}$ and $l_{e}(5.6 \mathrm{~cm}$ and $0.24 \mathrm{~cm}$, respectively, in Figures 2, 4, and 5) have been used for the calculation. A value of 22 has been used for $n$ for all phases.

with increasing applied frequency is observed. The equation is also able to mimic the observation of increased resolving power with decreasing OMS phase number. In addition to this, the equation, as with the experimental data, shows an increased rate of resolving power improvement (from the fundamental frequency to the overtone frequency corresponding to $h=2$ ) as a function of decreasing phase number. Finally, it should be noted that for the calculated resolving power, the rate of increase is greatest from the fundamental frequency to the overtone frequency corresponding to $h=2$. At that point, going from the $h=2$ to 3 overtone frequencies allowing ion transmission, the rate of increase is not as high. This occurs for each OMS phase system. Intuitively, this occurs because of the decrease in size of the transmitted ion beam portion (Figure 3). While this portion of the beam is decreasing with increasing overtone frequency, the overall ion transit time is not. Thus, diffusion becomes more significant for this shorter transmitted beam. Because, diffusion is overestimated in our resolving power equation (as the maximum expected value), the theoretical values drop below the experimental values.

\section{Evaluation of the Resolving Power Equation: Dependence on Diffusion}

The OMS resolving power equation can be used to begin to evaluate factors involving ion diffusion and their influence on the overall attainable resolving power. The resolving power has been evaluated as a function of two parameters from eq 1 that affect the degree of ion diffusion; these are $E$ and $T$. Here we note that from our treatment of $L\left[n \cdot\left(l_{t}+l_{e}\right)\right]$, we observed a negligible effect from diffusion over the experimental range of L (Supplementary Figure 3). For the determination of the effect of $E$ and $T$, a comparison of the $m=$ 1,3 , and 5 harmonic frequencies for a two-phase system has been carried out. The overall change for both parameters is relatively small across reasonable instrumentation operating ranges and is most pronounced for the $m=5$ frequency. For example, from $E=7 \mathrm{~V} \cdot \mathrm{cm}^{-1}$ to $\mathrm{E}=25 \mathrm{~V} \cdot \mathrm{cm}^{-1}$ only an $\sim 39 \%$ improvement in resolving power is observed; similarly, the range of $T=$ 200 to $400 \mathrm{~K}$ exhibits only an $\sim 29 \%$ improvement in resolving power (going from high to low temperatures). This analysis serves as a nice double check of the resolving power equation. That is, the boundaries for improved resolving power are the same as those expected from IMS measurements. A difference is the limited effect of ion diffusion on overall resolving power for OMS experiments (under current operating conditions). The effects of $E$ and $T$ can be observed in Supplementary Figure 4 in the Supplementary Information section.

Although, diffusion does not appear to affect the resolving power significantly, it is useful to consider a possible explanation for the observation of a larger change at higher overtone numbers. As mentioned above, the transmitted ion beam portions are much smaller for the higher overtones (Figure 3). The contribution of diffusion becomes more significant for these shorter ion beam portions (note the overall transit time is not changed). That is, the distance traveled due to diffusion (counter to the mobility of the ion for the OMS resolving power equation) is a larger percentage of the overall transmission region size.

Another factor that influences the degree of peak broadening due to diffusion is the ratio of the transmit and elimination $\left(l_{t}\right.$ and $\left.l_{e}\right)$ region length. To assess the effect of these lengths on computed resolving power, each is held constant while the other is varied. Four preliminary studies, summarized in Table 1 , were carried out. The first involves the changing of the $l_{e}$ region while maintaining a constant value for $l_{t}$. In general, the resolving power increases for increasing $l_{t}$. This result again suggests that peak broadening due to diffusion is worse for smaller ion beam portions as we have discussed above. The next study involves the maintaining of the combined length $\left(l_{e}+l_{t}\right)$, while changing the ratio of the two regions. That is, in one instance one region is much greater than the other and then the distances are reversed. Increasing the length of the $l_{e}$ region has a much greater impact in improving resolving power. Upon observation of the equation this becomes clearer. As $l_{e}$ increases, the term containing $n$ becomes larger than $(n-1) / n$. That is the difference in pulse widths at the base and apex of the peak in the OMS spectrum 
(Figure 4) becomes smaller leading to an increase in resolving power. The fourth study involves computing $R_{O M S}$ for equi-distant $l_{t}$ and $l_{e}$ regions, which does not significantly impact $R_{\text {OMS }}$ except when compared with instances, where $l_{e}$ is significantly smaller. The final study involves changing the $l_{e}$ region while maintaining the $l_{t}$ region. Here only a modest increase in resolving power is achieved. Based on the above studies, apparently by increasing $l_{e}$ substantially, a significant improvement in resolving power can be obtained. However, in considering the origin of the ions that limit resolving power, the only method by which ions of the same mobility arrive at drastically different positions in the OMS device over the same time period is due to diffusion. Therefore, there is a limit to the size of the $l_{e}$ region after which ion transmission would be blocked. Additionally, a larger $l_{e}$ leads to a decrease in experimental duty cycle.

The computed values in Table 2 show that the resolving power equation can be used to rapidly test the separation efficiency of specific instrumental configurations. An understanding of the trends for each of the variables suggests the direction in which one should pursue higher resolving power. Another interesting feature of the table is that a relatively high resolving power $(\sim 85)$ can be obtained with a fairly small device.

\section{The Use of OMS for Structural Analysis}

Until now the discussion has focused on the ability to estimate $R_{O M S}$ for a given OMS experimental setup. Briefly, the discussion has touched upon the application of OMS as a mobility selection device. As a final thought, an application of ion structure analysis is discussed. As mentioned above, the measurement of $K$ by IMS can be used to make inferences about the shapes of specific ions. This is accomplished by using measured values of $K$ to determine a collision cross section from [1]

$$
\Omega=\frac{(18 \pi)^{1 / 2}}{16} \frac{z e}{\left(k_{b} T\right)^{1 / 2}}\left[\frac{1}{m_{I}}+\frac{1}{m_{B}}\right]^{1 / 2} \frac{t_{D} E}{L} \frac{760}{P} \frac{T}{273.2} \frac{1}{N^{\prime}}
$$

where $P$ and $N$ correspond to the buffer gas pressure and

Table 2. Calculated $R_{O M S}$ for different values for $l_{t}$ and $l_{e}$

\begin{tabular}{rlcccc}
\hline \multicolumn{2}{c}{ Dimensions } & & \multicolumn{3}{c}{$R_{\text {OMS }}$} \\
\cline { 5 - 6 }$I_{t}(\mathrm{~cm})$ & $l_{e}(\mathrm{~cm})$ & & $m=1$ & $m=3$ & $m=5$ \\
\hline \hline 1.4 & 0.24 & & 17.0 & 30.0 & 35.4 \\
2.8 & 0.24 & 17.7 & 34.6 & 42.7 \\
5.6 & 0.24 & & 18.5 & 39.5 & 51.1 \\
11.2 & 0.24 & 19.2 & 44.4 & 60.0 \\
1.4 & 5.6 & & 52.6 & 76.9 & 84.7 \\
2.5 & 2.5 & 29.2 & 51.7 & 61.2 \\
5.6 & 1.4 & 21.8 & 45.4 & 58.1 \\
5.6 & 0.12 & 18.2 & 38.8 & 50.3 \\
5.6 & 0.24 & 18.5 & 39.5 & 51.1 \\
5.6 & 0.48 & 19.2 & 40.7 & 52.6 \\
\hline
\end{tabular}

the neutral number density under STP conditions, respectively. The variables $m_{I}$ and $m_{B}$ correspond to the mass of the ion and the mass of the buffer gas, respectively. The variables $E, L, P$, and $t_{D}$ can be measured accurately providing high accuracy cross section determinations.

To obtain a collision cross section from an OMS measurement, the distance the ion travels in a specified amount of time must be determined. From an OMS distribution this translates into ion displacement over the time equal to the inverse of the peak apex frequency. For the fundamental frequency, recalling that ion displacement corresponds to one $d$ region, the calculation is relatively straightforward. For conditions that employ overtone frequencies $(m>1)$, recall that ion displacement is equal to a fraction of the $d$ region (or $d / m$, see Figure 3). Additionally, because $m$ can be expressed in terms of $\Phi$ and $h$ [i.e., $m=\Phi(h-1)+1$ ], it becomes possible to rewrite eq 9 for OMS measurements as,

$$
\begin{aligned}
\Omega= & \frac{(18 \pi)^{1 / 2}}{16} \frac{z e}{\left(k_{b} T\right)^{1 / 2}}\left[\frac{1}{m_{I}}+\frac{1}{m_{B}}\right]^{1 / 2} \\
& \times \frac{E[\phi(h-1)+1]}{f\left(l_{t}+l_{e}\right)} \frac{760}{P} \frac{T}{273.2} \frac{1}{N} .
\end{aligned}
$$

One concern about the measurement of cross sections in a device that uses electrodynamic fields is the effect of the voltage slewing rate on the overall measurement. Experiments have shown that $\sim 20$ ns are required to achieve stable fields after switching. From a number of experiments, for a variety of ions (different charge states of ubiquitin, peptides, and carbohydrates), the cross sections obtained from OMS measurements differ from those obtained by IMS techniques by $2 \%$ to $4 \%$ on average. Noting that this difference is larger than the errors obtained for different IMS instruments (typically $<2 \%$ ), a portion may indeed result from field inhomogeneity. It may also result from slight errors in the determination of drift region segment length. The propagation of such an error would be more problematic than a small error in the overall length of a drift tube for an IMS instrument.

The ability to determine accurate collision cross sections may serve a number of applications. One example may include aiding in the identification of mixture components. That is, with significant enhancements to component resolution, cross sections determined for features in OMS distributions can be compared against trial structures [42, 50-62]. Such an approach could be used to select specific structures for further analysis.

\section{Summary}

Simulations involving the transport of ions through multiple drift regions have been compared with experimental data to develop a better understanding of the origin of resolving power in OMS. The results lead to an 
expression for resolving power that includes experimental variables associated with ion diffusion $(E, L$, and T) as well as variables related to OMS instrument operation $\left(\Phi, n, f, I_{t}\right.$, and $\left.I_{e}\right)$. The impact of the various parameters on resolving power was described. A surprising result of the current study is the unit proportionality observed for the number of drift regions and the overall frequency. This observation is distinguished from that for conditions affecting the resolving power of traditional IMS techniques in that, for $R_{O M S}$, greater improvements in resolving power are provided per percent change in parameters affecting resolving power. Overall, this understanding suggests that it should be possible to build instruments with relatively high resolving power that are capable of transmitting ions with well defined mobilities.

\section{Acknowledgments}

The authors acknowledge support in part for the development of new instrumentation by a grants from the National Institutes of Health (AG-024547-01 and P41-RR018942), and the METACyte initiative funded by a grant from the Lilly Endowment. The authors are grateful for numerous stimulating discussions with their colleagues, Liang-shi Li, Caroline C. Jarrold, and Gary M. Hieftje about overtones and instrumentation in general. They also thank John Poehlman and Andrew Alexander for technical support.

\section{Appendix A Supplementary Material}

Supplementary material associated with this article may be found in the online version at doi:10.1016/ j.jasms.2009.01.001.

\section{References}

1. Mason, E. A.; McDaniel, E. W. Transport Properties of Ions in Gases; Wiley: New York, 1988; p. 137-223.

2. St. Louis, R. H.; Hill, H. H. For a review of IMS techniques see: Ion Mobility Spectrometry in Analytical Chemistry. Crit. Rev. Anal. Chem. 1990, 21, 321 (and references therein).

3. Clemmer, D. E.; Jarrold, M. F. For a review of IMS techniques see: Ion Mobility Measurements and Their Applications to Clusters and Biomolecules. J. Mass Spectrom. 1997, 32, 577-592, and references therein.

4. Hoaglund Hyzer, C. S.; Counterman, A. E.; Clemer, D. E. For a review of IMS techniques see: Anhydrous Protein Ions. Chem. Rev. 1999, 99, 3037-3079, and references therein.

5. Kemper, P. R.; Bowers, M. T. Electronic-State ChromatographyApplication to First-Row Transition-Metal Ions. J. Phys. Chem. 1991, 95, 5134-5146.

6. Revercomb, H. E.; Mason, E. A. Theory of Plasma Chromatography Gaseous Electrophoresis-Review. Anal. Chem. 1975, 47, 970-983.

7. Siems, W. F.; Wu, C.; Tarver, E. E.; Hill, H. H.; Larsen, P. R.; McMinn, D. G. Measuring the Resolving Power of Ion Mobility Spectrometers. Anal. Chem. 1994, 66, 4195.

8. Tang, K.; Li, F.; Shvartburg, A. A.; Stritmatter, E. F.; Smith, R. D. Two-Dimensional Gas-Phase Separations Coupled to Mass Spectrometry for Analysis of Complex Mixtures. Anal. Chem. 2005, 77, 6381-6388.

9. Dougard, P.; Hudgins, R. R.; Clemmer, D. E.; Jarrold, M. F. HighResolution Ion Mobility Measurements. Rev. Sci. Instrum. 1997, 119, 2240

10. Wu, C.; Siems, W. F.; Asbury, G. R.; Hill, H. H. Electrospray Ionization High-Resolution Ion Mobility Spectrometry-Mass Spectrometry. Anal. Chem. 1998, 70, 4929-4938.

11. Srebalus, C. A.; Li, J.; Marshall, W. S.; Clemmer, D. E. Gas-Phase Separations of Electrosprayed Libraries. Anal. Chem. 1999, 71, 39183927.

12. Tang, K.; Shvartsburg, A. A.; Lee, H. N.; Prior, D. C.; Buschbach, M. A.; Li, F.; Tolmachev, A.; Anderson, G. A.; Smith, R. D. High-Sensitivity Ion Mobility Spectrometry/Mass Spectrometry Using Electrodynamic Ion Funnel Interfaces. Anal. Chem. 2005, 77, 3330-3339.
13. Merenbloom, S. I.; Koeniger, S. L.; Bohrer, B. C.; Valentine, S. J.; Clemmer, D. E. Improving the Efficiency of IMS-IMS by a Combing Technique. Anal. Chem. 2008, 80(6), 1918-1927.

14. Shvartsburg, A. A.; Smith, R. D. Scaling the Resolving Power and Sensitivity for Planar FAIMS and Mobility-Based Discrimination in Flow- and Field-Driven Analyzers. J. Am. Soc. Mass Spectrom. 2007, 18, 1672-1681.

15. Carnahan, B.; Day, S.; Kouznetsov, V.; Matyjaszczuk, M.; Tarassov, A. Field Ion Spectrometry-A New Analytical Technology for Trace Gas Analysis. Proceedings of the International Conference on Advance in Instrumentation and Control ISA; 1996; p. 87-95.

16. Purves, R. W.; Guevremont, R.; Day, S.; Pipich, C. W.; Matyjaszcayk, M. S Mass Spectrometric Characterization of a High-Field Asymmetric Waveform Ion Mobility Spectrometer. Rev. Sci. Instrum.; Chicago, IL, October 1998, 69, 4094.

17. Purves, R. W.; Guevremont, R. Electrospray Ionization High-Field Asymmetric Waveform Ion Mobility Spectrometry-Mass Spectrometry. Anal. Chem. 1999, 71, 2346-2357.

18. Eiceman, G. A.; Tadjikov, B.; Krylov, E.; Nazarov, E. G.; Miller, R. A. Westbrook, J.; Funk, P. Miniature Radio-Frequency Mobility Analyzer as a Gas Chromatographic Detector for Oxygen-Containing Volatile Organic Compounds, Pheromones, and Other Insect Attractants. J. Chromatogr. A 2001, 917, 205-217.

19. Venne, K.; Bonneil, E.; Eng, K.; Thibault, P. Improvement in Peptide Detection for Proteomics Analyses Using NanoLC-MS and High-Field Asymmetry Wafeform Ion Mobility Mass Spectrometry. Anal. Chem. 2005, 77, 2176-2186.

20. Barnett, D. A.; Purves, R. W.; Ells, B.; Guevremont, R. Separation of o-, m-, and p-Phthalic Acids by High-Field Asymmetric Waveform Ion Mobility Spectrometry (FAIMS) Using Mixed Carrier Gases. J. Mass Spectrom. 2000, 35(8), 976-980.

21. Guevremont, R.; Purves, R. Comparison of Experimental and Calculated Peak Shapes for Three Cylindrical Geometry FAIMS Prototypes of Differing Electrode Diameters. J. Am. Soc. Mass Spectrom. 2005, 16(3), 349-362.

22. Guevremont, R.; Thekkadath, G.; Hilton, C. K. Compensation Voltage (CV) Peak Shapes Using a Domed FAIMS with the Inner Electrode Translated to Various Longitudinal Positions. J. Am. Soc. Mass Spectrom. 2005, 16(6), 948-956.

23. Rossell-Llompart, J.; Loscertales, I. G.; Bingham, D.; de la Mora, J. F. Sizing Nanoparticles and Ions with a Short Differential Mobility Analyzer. J. Aerosol Sci. 1996, 27(5), 695-719.

24. Labowsky, M.; de la Mora, J. F. Novel Ion Mobility Analyzers and Filters. J. Aerosol Sci. 2006, 37(3), 340-362.

25. McMurry, P. H. For a recent review regarding the history of DMA for analysis of particles see: A Review of Atmospheric Aerosol Measurements. Atmos. Environ. 2000, 34, 1959-1999, and references therein.

26. Wittmer, D.; Luckenbill, B. K.; Hill, H. H.; Chen, Y. H. ElectrosprayIonization Ion Mobility Spectrometry. Anal. Chem. 1994, 66, 2348-2355.

27. Clemmer, D. E.; Hudgins, R. R.; Jarrold, M. F. Naked Protein Conformations-Cytochrome $c$ in the Gas Phase. J. Am. Chem. Soc. 1995, 117, 10141-10142.

28. von Helden, G.; Wyttenbach, T.; Bowers, M. T. Conformation of Macromolecules in the Gas Phase-Use of Matrix-Assisted LaserDesorption Methods in Ion Chromatography. Science 1995, 267, 14831485.

29. von Helden, G.; Wyttenbach, T.; Bowers, M. T. Inclusion of a MALDI Ion-Source in the Ion Chromatography Technique-Conformational Information on Polymer and Biomolecular Ions. Int. J. Mass Spectrom. Ion Processes 1995, 146, 349-364.

30. Chen, Y. H.; Siems, W. F.; Hill, H. H. Jr. Fourier Transform Electrospray Ion Mobility Spectrometry. Anal. Chim. Acta 1996, 334(1/2), 75-84

31. Gillig, K. J.; Ruotolo, B.; Stone, E. G.; Russell, D. H.; Fuhrer, K.; Gonin, M.; Schultz, A. J. Coupling High-Pressure MALDI with Ion Mobility/ Orthogonal Time-of-Flight Mass Spectrometry. Anal. Chem. 2000, 72, 3965-3971.

32. Hoaglund, C. S.; Valentine, S. J.; Sporleder, C. R.; Reilly, J. P.; Clemmer, D. E. Three-Dimensional Ion Mobility/TOFMS Analysis of Electrosprayed Biomolecules. Anal. Chem. 1998, 70, 2236-2242.

33. Hoaglund-Hyzer, C. S.; Li, J.; Clemmer, D. E. Mobility Labeling for Parallel CID of Ion Mixtures. Anal. Chem. 2000, 72, 2737-2740.

34. Bluhm, B. K.; Gillig, K. J.; Russell, D. H. Development of a FourierTransform Ion Cyclotron Resonance Mass Spectrometer-Ion Mobility Spectrometer. Rev. Sci. Instrum. 2000, 71(11), 4078-4086.

35. Hoaglund-Hyzer, C. S.; Clemmer, D. E. Ion Trap/Ion Mobility/ Quadrupole/Time-of-Flight Mass Spectrometry for Peptide Mixture Analysis. Anal. Chem. 2001, 73, 177-184.

36. Counterman, A. E.; Clemmer, D. E. Large Anhydrous Polyalanine Ions: Evidence for Extended Helices and Onset of a More Compact Structure. J. Am. Chem. Soc. 2001, 123, 1490-1498.

37. Valentine, S. J.; Kulchania, M.; Barnes, C. A. S.; Clemmer, D. E. Multidimensional Separations of Complex Peptide Mixtures: A Combined HighPerformance Liquid Chromatography/Ion Mobility/Time-of-Flight Mass Spectrometry Approach. Int. J. Mass Spectrom. 2001, 212, 97-109.

38. Myung, S.; Badman, E.; Lee, Y. J.; Clemmer, D. E. Structural Transition of Electrosprayed Ubiquitin Ions Stored in an Ion Trap Over $\sim 10 \mathrm{~ms}$ to 30 s. J. Phys. Chem. A 2002, 106, 9976-9982.

39. Tang, K.; Shvartsburg, A. A.; Lee, H. N.; Prior, D. C.; Buschbach, M. A.; Li, F. M.; Tolmachev, A. V.; Anderson, G. A.; Smith, R. D. High- 
Sensitivity Ion Mobility Spectrometry/Mass Spectrometry Using Electrodynamic Ion Funnel Interfaces. Anal. Chem. 2005, 77, 3330-3339.

40. Clowers, B. H.; Siems, W. F.; Hill, H. H.; Massick, S. M. Hadamard Transform Ion Mobility Spectrometry. Anal. Chem. 2006, 78(1), 44-51.

41. Sun, W. J.; May, J. C.; Russell, D. H. A Novel Surface-Induced Dissociation Instrument for Ion Mobility-Time-of-Flight Mass Spectrometry. Int. J. Mass Spectrom. 2007, 259(1/3), 79-86.

42. Mack, E. Average Cross-Sectional Areas of Molecules by Gaseous Diffusion Methods. J. Am. Chem. Soc. 1925, 47, 2468-2482.

43. Shvartsburg, A. A.; Jarrold, M. F. An Exact Hard-Spheres Scattering Model for the Mobilities of Polyatomic Ions. Chem. Phys. Lett. 1996, 261, 86-91.

44. Mesleh, M. F.; Hunter, J. M.; Shvartsburg, A. A.; Schatz, G. C.; Jarrold, M. F. Structural Information from Ion Mobility Measurements: Effects of the Long-Range Potential. J. Phys. Chem. 1996, 100, 16082-16086.

45. Wyttenbach, T.; von Helden, G.; Batka, J. J.; Carlat, D.; Bowers, M. T. Effect of the Long-Range Potential on Ion Mobility Measurements. J. Am. Soc. Mass Spectrom. 1997, 8, 275-282.

46. Shvartsburg, A. A.; Hudgins, R. R.; Dugourd, P.; Jarrold, M. F. Structural Information from Ion Mobility Measurements: Applications to Semiconductor Clusters. Chem. Soc. Rev. 2001, 30, 26-35.

47. Julian, R. R.; Mabbett, S. R.; Jarrold, M. F. Ion Funnels for the Masses: Experiments and Simulations with a Simplified Ion Funnel. J. Am. Soc. Mass Spectrom. 2005, 16(10), 1708-1712.

48. Koeniger, S. L.; Merenbloom, S. I.; Valentine, S. J.; Jarrold, M. F.; Udseth, H.; Smith, R. D.; Clemmer, D. E. An IMS-IMS Analogue of MS-MS. Anal. Chem. 2006, 68, 4161-4174.

49. Dahl, D. A. SIMION (Version 7.0); Idaho National Engineering Laboratory: Idaho Falls, ID.

50. Mack, E. Average Cross-Sectional Areas of Molecules by Gaseous Diffusion Methods. J. Am. Chem. Soc. 1925, 47, 2468-2482.

51. von Helden, G.; Hsu, M.-T.; Kemper, P. R.; Bowers, M. T. Structures of Carbon Cluster Ions from 3 to 60 Atoms-Linears to Rings to Fullerenes. J. Chem. Phys. 1991, 95(5), 3835-3837.
52. Jarrold, M. F.; Constant, V. A. Silicon Cluster Ions-Evidence for a Structural Transition. Phys. Rev. Lett. 1991, 67(21), 2994-2997.

53. von Helden, G.; Hsu, M.-T.; Gotts, N.; Bowers, M. T. Carbon Cluster Cations with up to 84 Atoms-Structures, Formation Mechanism, and Reactivity. J. Phys. Chem. 1993, 97, 8182-8192.

54. Shelimov, K. B.; Hunter, J. M.; Jarrold, M. F. Small Carbon RingsDissociation, Isomerization, and a Simple Model Based on Strain. Int. J. Mass Spectrom. Ion Phys. 1994, 138, 17-31.

55. Clemmer, D. E.; Hudgins, R. R.; Jarrold, M. F. Ion Mobility Measurements and Their Applications to Clusters and Biomolecules. J. Am. Chem. Soc. 1995, 117, 10141-10142.

56. Mesleh, M. F.; Hunter, J. M.; Shvartsburg, A. A.; Schatz, G. C.; Jarrold M. F. Structural Information from Ion Mobility Measurements: Effects of the Long-Range Potential. J. Phys. Chem. 1996, 100, 16082-16086.

57. Wyttenbach, T.; von Helden, G.; Batka, J. J.; Carlat, D.; Bowers, M. T. Effect of the Long-Range Potential on Ion Mobility Measurements. J. Am. Chem. Soc. 1997, 8, 275-282.

58. Shvartsburg, A. A.; Hudgins, R. R.; Dugourd, P.; Jarrold, M. F. Structural Information from Ion Mobility Measurements: Applications to Semiconductor Clusters. Chem. Soc. Rev. 2001, 30, 26-35.

59. Valentine, S. J.; Anderson, J. G.; Ellington, A. D.; Clemmer, D. E. Disulfide-Intact and -Reduced Lysozyme in the Gas Phase: Conformations and Pathways of Folding and Unfolding. J. Phys. Chem. B 1997, 101, 3891-3900.

60. Hudgins, R. R.; Ratner, M. A.; Jarrold, M. F. Design of Helices that are Stable In Vacuo. J. Am. Chem. Soc. 1998, 120, 12974-12975.

61. Counterman, A. E.; Clemmer, D. E. Large Anhydrous Polyalanine ions: Evidence for Extended Helices and Onset of a More Compact State. J. Am. Chem. Soc. 2001, 123, 1490-1498.

62. Myung, S.; Badman, E.; Lee, Y. J.; Clemmer, D. E. Structural Transitions of Electrosprayed Ubiquitin Ions Stored in an Ion Trap Over $\sim 10 \mathrm{~ms}$ to 30 s. J. Phys. Chem. A 2002, 106, 9976-9982. 